\title{
Correlation between the surface defect distribution and minority carrier transport properties in GaN
}

\author{
P. M. Bridger, Z. Z. Bandić, E. C. Piquette, and T. C. McGill ${ }^{\text {a) }}$ \\ Thomas J. Watson, Sr. Laboratory of Applied Physics, 128-95, California Institute of Technology, \\ Pasadena, California 91125
}

(Received 20 May 1998; accepted for publication 6 October 1998)

\begin{abstract}
We have studied linear dislocations and surface defects in $p$ - and $n$-type metalorganic chemical vapor deposition, hydride vapor phase epitaxy, and molecular beam epitaxy grown GaN films on sapphire with atomic force microscopy. The surface pits due to threading dislocations were found not to be distributed randomly but on the boundaries of growth columns. The dislocations are thought to be electrically active since the average distance between them (average column size) is comparable to minority carrier diffusion lengths as measured by electron beam induced current experiments on Schottky diodes fabricated with the same material. Diffusion lengths found for holes and electrons are on the order of $L_{p}=0.28 \mu \mathrm{m}$ and $L_{e}=0.16 \mu \mathrm{m}$ which corresponded to the sizes of regions free from surface dislocations in both cases and can be described by a simple model of recombination on grain boundaries. (C) 1998 American Institute of Physics.
\end{abstract}

[S0003-6951(98)04649-X]

Gallium nitride based devices are currently of great interest for optoelectronic ${ }^{1-3}$ as well as high power and high temperature electronics. ${ }^{4,5}$ Since many of the performance characteristics of bipolar devices are determined by the minority carrier transport properties (diffusion lengths and lifetimes), ${ }^{6}$ it is useful to determine how they are affected by the defect structure. It has been assumed that linear dislocations were electrically inert ${ }^{7-9}$ since they had little effect on the performance or reliability of $\mathrm{GaN}$ light emitting diodes. However, the correlation between cathodoluminescence (CL) and surface morphology (dislocations terminating as pits in the surface) shown by Rosner et al. indicates that linear dislocations are important in recombination processes. ${ }^{10}$

In this experiment, we have determined both electron and hole diffusion lengths from electron beam induced current (EBIC) measurements on material that was characterized by atomic force microscopy (AFM). We then compare the distribution of defect induced depressions on the surface with the measured diffusion lengths. Because the density of linear dislocations threading the $\mathrm{GaN}$ is believed to be approximately the same as those reaching the top surface of the film for thin films, ${ }^{7}$ the two-dimensional (2D) AFM data should represent the $3 \mathrm{D}$ distribution of those dislocations. For thicker films, however, the density of threading dislocations reaching the surface of the film decreases with film thickness. Moreover, the AFM does not reveal all types of defects. Nevertheless, it is the nonuniform distribution of the defects and not their number that is considered here.

The AFM data were obtained using a Digital Instruments Nanoscope IIIa operating in tapping mode. A cross check of the data was performed using contact AFM with oxide sharpened tips and no significant differences were found. The EBIC experiments were conducted in a JEOL6400 V electron microscope with a GW Electronics preamplifier followed by GW Electronics specimen current amplifier or Kei-

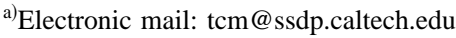

thley 486 picoammeter. The entire apparatus was enclosed in an electromagnetic interference (EMI) cancellation cage. Gold and $\mathrm{Ti} / \mathrm{Al} / \mathrm{Ni} / \mathrm{Au}$ were used as the Schottky and ohmic contacts in the case of $n$-type $\mathrm{GaN}$ while $\mathrm{Ni} / \mathrm{Au}$ and $\mathrm{Ti} / \mathrm{Au}$ were used in the $p$-type case. For a more detailed discussion of the EBIC measurements see Refs. 6 and 11 and in general Ref. 12. A sample of the EBIC data is shown in Fig. 1. The profile of the EBIC as a function of distance from the edge of the Schottky contact is fitted to the theoretical relation $I$ $=k x^{-1 / 2} \exp (-x / L)$ to determine the diffusion length.

The $n$-type metalorganic chemical vapor deposition (MOCVD) films for this study were commercially grown unintentionally doped $2-\mu \mathrm{m}$-thick $\mathrm{GaN}$ on sapphire with a carrier concentration in the $10^{17} \mathrm{~cm}^{-3}$ range. The $p$-type MOCVD films were commercially grown, Mg doped $2-\mu \mathrm{m}-$ thick $\mathrm{GaN}$ on sapphire with a carrier concentration in the $10^{17} \mathrm{~cm}^{-3}$ range. The hydride vapor phase epitaxy (HVPE) sample was $10-\mu \mathrm{m}$-thick GaN grown on sapphire. The 10 $\mu \mathrm{m}$ layer consisted of an 8 - $\mu \mathrm{m}$-thick low conductivity $\left(\approx 10^{16} \mathrm{~cm}^{-3}\right.$ ) layer and a highly conductive bottom layer.

The molecular beam epitaxy (MBE) sample was grown on sapphire using a thin AlN buffer layer and a rf plasma

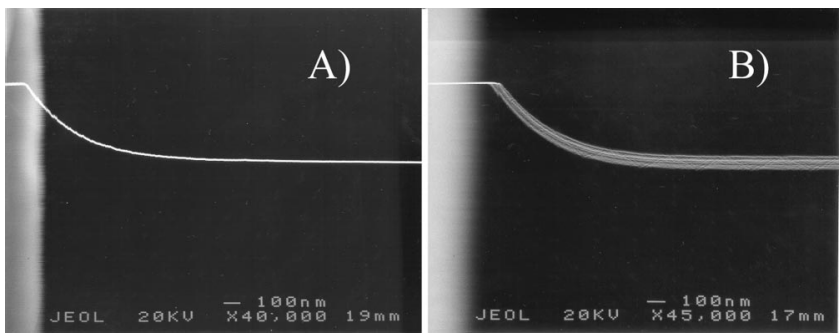

FIG. 1. Line scan profiles of the EBIC superimposed on the secondary electron image of the Schottky contact. (A) p-type MOCVD grown GaN. The beam current was $0.5 \mathrm{nA}$ at an accelerating voltage of $20 \mathrm{kV}$; (B) n-type MOCVD grown GaN. The beam current was $1.06 \mathrm{nA}$ at an accelerating voltage of $20 \mathrm{kV}$. The profile of the EBIC as a function of distance from the edge of the Schottky contact is fitted to the theoretical relation $I$ $=k x^{-1 / 2} \exp (-x / L)$ in order to determine the diffusion length. 


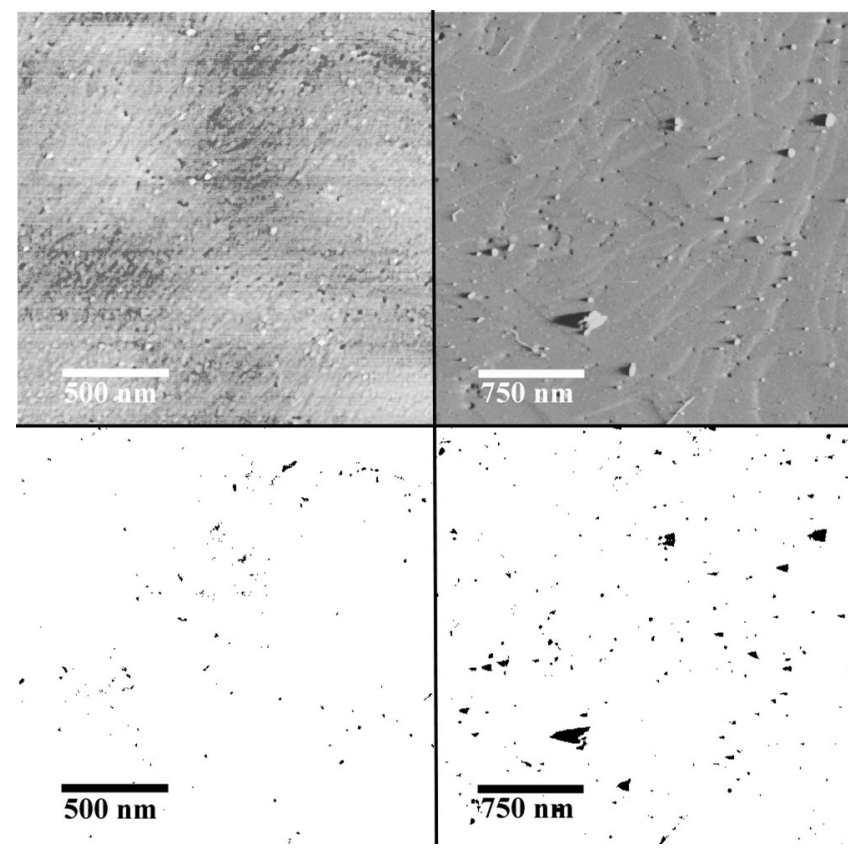

FIG. 2. Two samples of $n$-type MOCVD grown GaN. The top left-hand side image is a $3 \mu \mathrm{m}$ AFM scan and the top right-hand side image is a $2 \mu \mathrm{m}$ scan. Corresponding thresholded images are shown below to accentuate the defects which appear as 30-nm-dark areas. Their distribution does not appear to be random, but decorating the grain boundaries.

source for active nitrogen. The film was grown at $800{ }^{\circ} \mathrm{C}$, near stoichiometric conditions, to a thickness of $820 \mathrm{~nm}$. Layer thickness were confirmed by variable angle spectroscopic ellipsometry (VASE). The surface of the film shows hillocks which may be a result of gallium flux deficiency during growth or the presence of inversion domains due to the growth on bare sapphire.

Figure 2 shows representative results for $n$-type

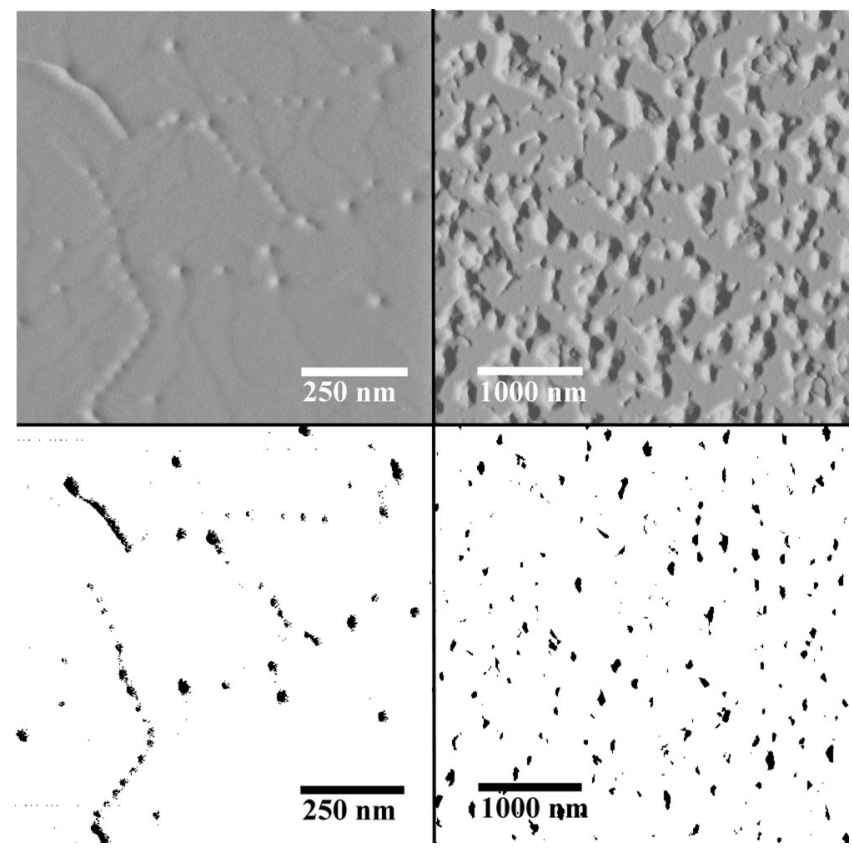

FIG. 3. Left-hand side: $1 \mu \mathrm{m}$ AFM image of $p$-type MOCVD grown GaN showing the pits on the surface from the termination of linear defects at the surface. Right-hand side: $4 \mu \mathrm{m}$ AFM image of $n$-type MBE grown $1 \mu \mathrm{m}$ thick GaN film on sapphire. The bottom left- and right-hand side images are the thresholded data to accentuate the distribution of the defects.

Downloaded 03 Apr 2006 to 131.215.225.171. Redistribution subject to AlP license or copyright, see http://apl.aip.org/apl/copyright.jsp

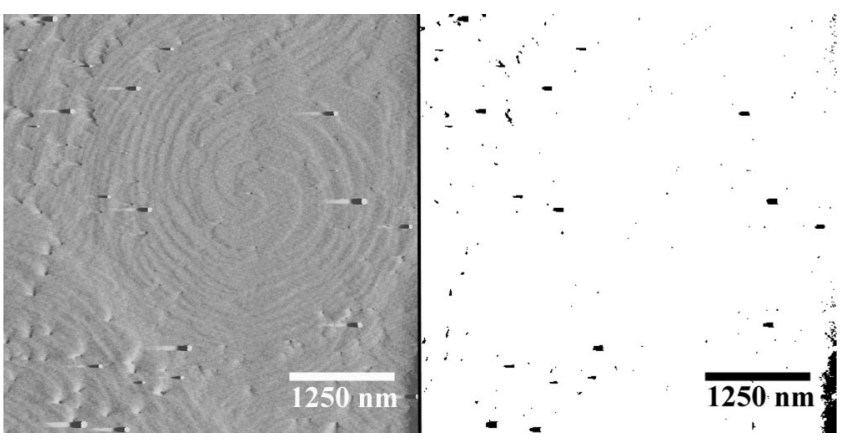

FIG. 4. Left-hand side: $5 \mu \mathrm{m}$ AFM image of $n$-type HVPE grown GaN showing the boundary between the two distinct regions. Right-hand side: the corresponding thresholded image to accentuate the defects.

MOCVD GaN. The small pits observed are $30 \mathrm{~nm}$ in size as was the case in Ref. 10. Although the two images are different (in one case the defects appear to lie along the grain boundaries and in the other terminating steps) the "defect free" regions are typically on the order of $0.5 \mu \mathrm{m}$ which also appears to be the case in Ref. 10. Grains of similar size have also been observed by scanning capacitance microscopy. ${ }^{13}$ The corresponding thresholded images are shown to accentuate the distribution of surface defects. The average measured hole diffusion lengths for both samples were the same to within experimental error; $L_{p}=0.28 \pm 0.02 \mu \mathrm{m}$. Figure 3 shows results for $p$-type MOCVD and $n$-type MBE grown $\mathrm{GaN}$ which also exhibited surface terminated defects. In the $p$-type sample (left-hand side) long trains of defects clearly outlined the grain boundaries which were in the same size range as the $n$-type material, $0.5 \mu \mathrm{m}$. Electron diffusion lengths in this $p$-type sample were measured to be $L_{e}=0.2$ $\pm 0.05 \mu \mathrm{m}$. The MBE film (right-hand side) clearly shows pyramidal hillocks associated with inversion domains that thread the GaN film. ${ }^{14}$ The flat regions between the hillocks are roughly $0.25 \mu \mathrm{m}$ in size and the hole diffusion length for this material was $L_{p}=0.22 \pm 0.03 \mu \mathrm{m}$. Figure 4 shows the $n$-type HVPE sample at a boundary between regions of flat and spiral growth. Very small surface pits are visible at the step boundaries in the spiral region whereas larger pits of the same size as those in Figs. 2 and 3 are visible in the flat region. A boundary between the two regions is shown in Fig. 4. Preliminary measurements of the hole diffusion length in this sample gave regions of higher and lower $L_{p}$, which might correspond to the different growth regions which appear to have less and more surface defects as indicated by the right-hand side image in Fig. 4.

A summary of the EBIC results is shown in Table I. We can notice that the minority carrier diffusion lengths are al-

TABLE I. Summary of the EBIC measurements on several GaN samples. The second column labeled with $C C$ represents carrier concentration. The diffusion lengths in each row of the table are averages over a range of beam currents and different measurement positions on the sample. The last column labeled with $D D$ gives density of linear dislocations for each sample.

\begin{tabular}{lccc}
\hline \multicolumn{1}{c}{ Sample } & $C C\left(\mathrm{~cm}^{-3}\right)$ & Diffusion length & $D D\left(\mathrm{~cm}^{-2}\right)$ \\
\hline$n$-MOCVD & $10^{17}$ & $L_{p}=0.28 \pm 0.02 \mu \mathrm{m}$ & $(2-5) \times 10^{9}$ \\
$n$-MBE & $10^{16}$ & $L_{p}=0.22 \pm 0.03 \mu \mathrm{m}$ & $10^{9}$ \\
$n$-HVPE & $10^{16}$ & $L_{p}>2 \mu \mathrm{m}$ & $10^{8}$ \\
$p$-MOCVD & $10^{17}$ & $L_{n}=0.20 \pm 0.05 \mu \mathrm{m}$ & $5 \times 10^{9}$ \\
\hline \hline
\end{tabular}


ways smaller than either the average grain size or average column size delineated by inversion domains. A simple model can be used to explain this observation. By solving the 1D diffusion equation for minority hole recombination on periodic grain boundaries (due to defects or inversion domains) in a manner similar to Ref. 15 we have

$$
D \frac{d^{2} p}{d x^{2}}=\frac{\partial p}{\partial t},
$$

with the boundary condition $p\left(m^{*} d\right)=0$, where $m$ is an integer and $d$ is the distance between grain boundaries or columns separated by inversion domains. The boundary condition is set by the assumption that all the carriers recombine at the boundary. The separable solutions are of the form

$$
p(x, t)=e^{-t / \tau} p(x),
$$

where $p(x)=p_{0} \sin \left(n x / L_{p}\right)$. At the boundary, $p(m d)=0$ which gives $L_{p}=d / \pi$. Substituting measured values for $d$ $=0.5 \mu \mathrm{m}$ we find $L_{p} \approx 0.17 \mu \mathrm{m}$ which is in good agreement with the measured value.

In conclusion, structural characterization with AFM indicated that dislocations are not uniformly distributed and the size of the defect free regions are roughly the same size as the diffusion length of minority carriers in the material. We have measured both hole and electron diffusion lengths in MOCVD grown $\mathrm{GaN}$ and found them to be $L_{p}=0.28$ $\pm 0.03 \mu \mathrm{m}$ and $L_{e}=0.2 \pm 0.05 \mu \mathrm{m}$. We have also measured hole diffusion lengths in MBE and HVPE grown GaN. A nonuniform distribution of defects would offer one explanation why, on the assumption that defects are involved in recombination, a high "average" dislocation density would not necessarily yield small diffusion lengths.
This work was supported in part by the Defense Advanced Research Project under N00014-92-J-1845 and monitored by the Office of Naval Research. The authors would also like to thank J. Redwing, R. P. Vaudo, and V. M. Phanse of ATMI Inc. for the HVPE sample.

${ }^{1}$ S. Nakamura, M. Senoh, S. Nagahama, N. Iwasa, T. Yamada, T. Matsushita, Y. Sugimoto, and H. Kiyoku, Appl. Phys. Lett. 70, 1417 (1997).

${ }^{2}$ M. A. Kahn and M. S. Shur, Mater. Sci. Eng., B 46, 69 (1997).

${ }^{3}$ J. M. Van Hove, R. Hickman, J. J. Klaassen, P. P. Chow, and P. P. Ruden, Appl. Phys. Lett. 70, 2282 (1997).

${ }^{4}$ S. M. Mohammad and H. Morkoç, Prog. Quantum Electron. 20, 361 (1996).

${ }^{5}$ Y. F. Wu, B. P. Keller, S. Keller, N. X. Nguyen, M. Le, C. Nguyen, T. J. Jenkins, L. T. Kehias, S. P. DenBaars, and U. K. Mishra, IEEE Electron Device Lett. 18, 438 (1997).

${ }^{6}$ Z. Z. Bandić, P. M. Bridger, E. C. Piquette, T. F. Kuech, and T. C. McGill, Mater. Res. Soc. Symp. Proc. 483, 399 (1997).

${ }^{7}$ S. D. Lester, F. A. Ponce, M. G. Craford, and D. A. Steigerwald, Appl. Phys. Lett. 66, 1249 (1995).

${ }^{8}$ L. Sugiura, J. Appl. Phys. 81, 1633 (1997).

${ }^{9}$ F. A. Ponce, MRS Bull. 22, 51 (1997).

${ }^{10}$ S. J. Rosner, E. C. Carr, M. J. Ludowise, G. Girolami, and H. I. Erikson, Appl. Phys. Lett. 70, 420 (1997).

${ }^{11}$ Z. Z. Bandić, P. M. Bridger, E. C. Piquette, and T. C. McGill, Appl. Phys. Lett. 72, 3166 (1998).

${ }^{12}$ D. Chan, V. Ong, and J. Phang, IEEE Trans. Electron Devices 42, 963 (1995).

${ }^{13}$ P. J. Hansen, Y. E. Strausser, A. N. Erickson, E. J. Tarsa, P. Kozodoy, E. G. Brazel, J. P. Ibbetson, U. Mishra, V. Narayanamurti, S. P. DenBaars, and J. S. Speck, Appl. Phys. Lett. 72, 2247 (1998).

${ }^{14}$ L. T. Romano and T. H. Myers, Appl. Phys. Lett. 71, 3486 (1997).

${ }^{15}$ W. R. Harding, I. D. Blenkinsop, and D. R. Wright, Electron. Lett. 12, 502 (1976). 